\title{
Les marqueurs de l'éthylisme chronique. Focus sur les approches immuno-chimiques
}

\section{Chronic alcohol drinking and characterization using immuno-chemistry methods}

\author{
Pascal Kintz ${ }^{\star}$, Marion Villain, Ariane Mandel, Vincent Cirimele \\ Laboratoire ChemTox, 3 rue Gruninger, 67400 Illkirch, France
}

\begin{abstract}
Résumé - Les différents marqueurs biologiques de la consommation d'alcool éthylique peuvent être répartis en 2 groupes : marqueurs directs (éthanol, éthyl glucuronide, éthyl sulfate, esters éthyliques d'acides gras, cocaéthylène) et marqueurs indirects (VGM, $\gamma$-GT, ASAT, ALAT, CDT). Si les marqueurs directs sont essentiellement utiles pour caractériser une intoxication aigue, l'introduction du cheveu a permis d'augmenter la fenêtre de détection et les applications du dosage de l'éthyl glucuronide. Les marqueurs indirects sont des paramètres biologiques dont l'augmentation peut être la conséquence d'une consommation importante et régulière d'éthanol. L'objet de cette revue est de faire le point sur la pertinence de chaque test, en portant une attention particulière sur les approches immuno-chimiques.
\end{abstract}

Mots clés : Éthanol, addiction, éthylisme chronique, CDT, ethyl glucuronide

\begin{abstract}
This review deals with the 2 types of markers of ethyl alcohol consumption, including direct markers (ethanol, ethyl glucuronide, ethyl sulfate, fatty acid ethyl esters, cocaethylene) and indirect markers (MCV, $\gamma$-GT, ASAT, ALAT, CDT). For each test, sensitivity and specificity will be discussed. A special focus will be given to immunochemistry methods.
\end{abstract}

Key words: Ethanol, addiction, chronic alcohol drinking, CDT, ethyl glucuronide

Reçu le 19 novembre 2008, accepté après modifications le 5 mars 2009

Publication en ligne le 16 avril 2009

\section{Introduction}

Malgré une consommation en baisse depuis plusieurs décennies (9,3 litres d'alcool pur / habitant / an versus 12,4 en 1961), surtout chez les adultes, l'alcool éthylique reste la substance psychoactive la plus consommée en France. Moins de $10 \%$ des $12-75$ ans déclarent n'en avoir jamais bu. Le vin est l'alcool le plus consommé ( $77 \%$ en ont bu au moins une fois au cours de l'année), la consommation quotidienne augmentant avec l'âge (39\% des plus de 65 ans, contre $1 \%$ chez les moins de 20 ans). Les ivresses ponctuelles sont en revanche l'apanage des plus jeunes (binge drinking). Des disparités régionales de consommation ont été récemment mises en avant par l'Institut National de Prévention et d'Éducation pour la Santé (INPES). Chez les jeunes, la Bretagne est en tête pour la consommation, suivie de la Bourgogne et c'est l'Ile-de-France

\footnotetext{
^ Correspondance : Pascal Kintz, Tél. 0390400 540,
}

Fax. 0390400 541, pascal.kintz@wanadoo.fr qui ferme le ban. À l'âge adulte, les régions où on boit le plus tous les jours sont le Midi-Pyrénées, le Languedoc-Roussillon et le Nord-Pas de Calais [1].

En vrac, d'autres statistiques indiquent que la part des dépenses consacrées à l'alcool par les Français est de 8,9\% (en diminution, elle était de $12,4 \%$ en 1961). Il y a $13,7 \%$ de Français qui déclarent avoir consommé de l'alcool tous les jours de l'année. Enfin, si $58 \%$ de la consommation d'éthanol en France est sous forme de vin, $30 \%$ est de la bière, $7 \%$ des alcools forts et $5 \%$ du cidre (sources INSEE).

Sur la route, la tolérance zéro est en place en Hongrie, en République Tchèque, en Slovénie ou encore en Tunisie. La limite légale de l'alcoolémie reste très variable, passant de $0,1 \mathrm{~g} / \mathrm{L}$ en Algérie et en Guyane, à $0,4 \mathrm{~g} / \mathrm{L}$ en Chine, au Japon, en Norvège ou en Suède, puis $0,5 \mathrm{~g} / \mathrm{L}$ en Allemagne, en Australie, au Luxembourg ou aux Pays Bas et enfin à $0,8 \mathrm{~g} / \mathrm{L}$ aux États-Unis, au Royaume Uni ou en Irlande. Enfin, il n'y a pas de limite légale en Indonésie. 
En France, les problèmes liés à l'alcoolisme touchent environ 5 millions de personnes, dont environ 2 millions sont dépendantes. L'alcoolisme est la deuxième cause de mort évitable en France, après le tabac, avec 45000 décès par an (11000 cancers, 9000 cirrhoses, 2500 alcoolo-dépendances, et 22000 décès indirects liés à des troubles mentaux, cardiovasculaires et des accidents). Le syndrome d'alcoolisation fœtale touche chaque année entre 5 et 7000 nouveau-nés.

Le coût social de l'alcoolisme représente en France 17,4 milliards d'euros.

Dans ces conditions, il est important de disposer de tests biologiques, sensibles et spécifiques, permettant d'appréhender au mieux une consommation excessive d'éthanol. L'objet de cette revue est de faire le point sur les paramètres disponibles, en portant une attention particulière aux approches immuno-chimiques.

\section{Marqueurs direct}

\section{1 Éthanol}

De nombreuses revues ont fait le point sur les aspects les plus importants [2,3].

Il n'existe pas de méthode immuno-chimique permettant le dosage de l'éthanol sanguin. S'il est admissible en clinique d'utiliser une méthode enzymatique, il n'est pas prévu cette approche dans le cadre judiciaire en France (alors que d'autres pays l'acceptent comme l'Allemagne ou la Suisse). Les méthodes officielles de contrôle de l'alcoolémie sont la distillation selon Cordebard (arrêté du 27 septembre 1972, publié au J.O. du 30 novembre 1972) et la chromatographie en phase gazeuse (arrêté du 6 mars 1986, publié au J.O. du 16 mars 1986).

\section{2 Éthyl glucuronide}

Après administration, l'éthanol est essentiellement métabolisé par le foie (90-95\%), les reins $(0,5-2 \%)$, les poumons $(0,5-6 \%)$ et la peau $(0,5 \%)$ pour donner de l'eau et du gaz carbonique. Une très faible quantité d'éthanol (moins de $0,5 \%$ ) peut être éliminée sous forme d'éthyl glucuronide (EtG), un métabolite de phase II [4].

L'intérêt majeur de l'EtG est d'augmenter la fenêtre de détection de l'éthanol. En effet, on retrouve ce métabolite dans le sang alors que tout l'éthanol a déjà été éliminé [5]. Dans les urines, l'EtG est détectable jusqu'à 80 heures après exposition [6]. Les applications de la détermination de l'EtG sont nombreuses comme l'illustre sa caractérisation dans des matrices non conventionnelles, telles que les tissus, les os [7], la sueur [8] ou encore les cheveux [9].

L'aspect analytique est dominé par la chromatographie, soit gazeuse (GC-MS/NCI) soit liquide (LC-MS/MS). Dès 2002, Zimmer et coll. [10] proposait un test de dépistage par ELISA sur le sérum et les urines. Alors que les résultats sériques semblaient très encourageants, ceux obtenus dans les urines étaient décevants. Plus récemment, le test DRI-EtG EIA de Microgenics a été évalué dans les urines [11]. Selon les auteurs, en utilisant un seuil à $0,5 \mathrm{mg} / \mathrm{L}$, le kit a montré des capacités de sensibilité et de spécificité tout à fait acceptables, ce qui en ferait un excellent complément aux criblages des stupéfiants.

À ce jour, la caractérisation de l'EtG dans les cheveux constitue l'approche la plus pertinente pour la caractérisation de la consommation excessive de boisson alcoolisée [12]. Il n'existe pas encore d'harmonisation dans le seuil de positivité permettant de discriminer les buveurs dits sociaux des buveurs excessifs puisque la littérature mentionne des seuils entre 20 et $50 \mathrm{pg} / \mathrm{mg}$. Ce point fait actuellement l'objet de discussion au sein de la Society of Hair Testing et devrait déboucher en 2009 sur un consensus.

\section{3 Éthyl sulfate}

Tout comme l'EtG, l'éthyl sulfate (EtS) est un métabolite mineur de l'éthanol. Même si la fenêtre de détection se trouve allongée par rapport à l'éthanol, l'EtS ne présente que peu d'intérêt pratique par rapport à l'EtG [13]. Tout comme l'EtG, l'EtS apparaît comme particulièrement stable dans le temps, puisque ces 2 métabolites ont pu être retrouvés dans des prélèvements consécutifs à une exhumation après 27 ans [14].

Il n'existe pas de test immunochimique pour l'EtS.

\subsection{Esters éthyliques d'acides gras}

Les esters éthyliques d'acides gras (FAEE) sont un groupe d'une vingtaine de substances, comme l'éthyl laurate, l'éthyl myristate, l'éthyl palmitate ou encore l'éthyl stéarate. Ils sont formés en présence d'éthanol à partir d'acides gras libres, de triglycérides, de lipoprotéines ou de phospholipides par des FAEE synthases spécifiques, mais aussi par des carboxylestérases, des lipoprotéines lipases ou des cholesterol esterases. Dans le sang, les FAEE sont détectables 24 heures après l'arrêt de la consommation d'éthanol [15] et donc l'analyse pratique des FAEE est limitée à leur caractérisation dans les cheveux, la plupart du temps en complément de l'EtG [16]. Par contre, la caractérisation de l'alcoolisme fotal à partir des FAEE dans le méconium semble promise à un avenir certain, tant cette approche apparaît comme pertinente [17] puisqu'elle permet un recul sur environ 3 mois avant l'accouchement.

La chromatographie en phase gazeuse, couplée à la spectrométrie de masse est communément utilisée. Il n'existe pas de test immuno-chimique pour les FAEE.

\subsection{Cocaéthylène}

Produit de trans-estérification entre la cocaïne et l'éthanol, ce métabolite spécifique a surtout été étudié pour confirmer des résultats positifs de cocaïne tout en évitant le risque de contamination. Sa caractérisation en tant que marqueur d'une consommation d'alcool est très limitée et n'apporte aucune valorisation.

La chromatographie en phase gazeuse, couplée à la spectrométrie de masse est communément utilisée pour le dosage. Il n'existe pas de test immuno-chimique spécifique pour le cocaéthylène. 


\subsection{Phosphatidyléthanol}

Ce phospholipide est formé par action de la phospholipase D en présence d'éthanol. Il s'agit donc d'un marqueur spécifique, dont la normalisation est de l'ordre de 15 jours après l'abstinence. C'est un marqueur très utilisé en Suède pour discriminer dans le sang d'autopsie les consommateurs excessifs d'alcool [18] et qui semble, dans certains cas, plus pertinent que la mesure de la transferrine déficiente en carbohydrates [19]. Néanmoins, son dosage apparaît comme compliqué, faisant appel à de la chromatographie liquide.

\section{Marqueurs indirects}

\subsection{Marqueurs classiques de biochimie : VGM, $\gamma$-GT, ASAT, ALAT ...}

VGM, $\gamma$-GT, ASAT et ALAT sont des paramètres anciens, largement utilisés comme marqueurs de consommation et de surveillance de désintoxication. Deux informations conditionnent leur pertinence : spécificité et délai de normalisation après abstinence. Une revue complète et critique de la littérature a été publiée en 2006 sur ce sujet [20].

L'augmentation du volume globulaire moyen (VGM) au dessus de $98 \mathrm{fl}$ (normales entre 82 et $98 \mathrm{fl}$ ) constitue un argument en faveur d'une consommation chronique d'éthanol. Le VGM peut augmenter (macrocytose) chez certains patients qui ont une carence en vitamine B12 et/ou folates ou des troubles de la lignée érythroblastique. La spécificité est de l'ordre de 40 à $90 \%$ et la normalisation après abstinence se fait en 10 à 12 semaines.

La gamma glutamyl transférase $(\gamma-\mathrm{GT})$ est une enzyme inductible par l'éthanol mais aussi par les médicaments (barbituriques, phénytoïne, imipraminiques, antihypertenseurs, contraceptifs oraux... ). La $\gamma$-GT est élevée dans 35 à $90 \%$ des cas d'alcoolisme, mais aussi dans toutes les pathologies hépatobiliaires. L'abstinence permet un retour à la normale sous une quinzaine de jours.

Les aminotransférases, l'ASAT et l'ALAT sont des marqueurs d'une souffrance hépatique. Localisées dans les hépatocytes périportaux, leur élévation sérique traduit une altération de la membrane cellulaire. Pour la détection de l'éthylisme chronique, leur détermination est d'un intérêt limité.

L'ASAT est un mélange de 2 iso-enzymes, chacune étant codée par un gène distinct. L'iso-enzyme la plus importante est la mitochondriale (ASATm) dont l'activité représente environ $80 \%$ de l'activité aspartate amino-transférase totale (ASATt) au niveau hépatique; les $20 \%$ restants sont constitués par l'autre iso-enzyme, d'origine cytosolique (ASATc). Par contre, au niveau sérique, l'ASATm ne représente qu'une faible partie (moins de $10 \%$ ) de l'activité ASATt. Chez les alcooliques, la fraction sérique ASATm est augmentée (du fait de l'action toxique directe de l'éthanol sur les mitochondries) et il a été proposé que le rapport ASATm/ASATt puisse être un critère de discrimination entre une population de buveurs, qu'ils aient ou non une hépatopathie d'une population sobre, ayant un foie sain ou non [21]. Si ce test a fait ses preuves sur des patients hospitalisés, les conclusions semblent plus réservées sur la population générale. Le rapport ASATm/ASATt se normalise en 15 jours au cours de l'abstinence.

Enfin, la mesure de l'alpha gluthation S-transférase, augmentée lors de l'intoxication alcoolique aiguë ne semble pas pertinente pour mettre en évidence une atteinte hépatique liée à l'alcool [22].

La mesure de l'acétate sanguin, métabolite final de l'oxydation hépatique de l'alcool, permet en théorie de distinguer un alcoolisme chronique (augmentation de la concentration par induction enzymatique) d'une alcoolisation aiguë (qui n'affecte que très peu la concentration).

\subsection{Transferrine déficiente en carbohydrates}

La transferrine est une glycoprotéine synthétisée par le foie et impliquée dans le transport du fer. Elle possède 2 chaînes polysaccharidiques plus ou moins ramifiées (de 0 à 6) avec des résidus d'acide sialique. La forme tétrasialo-transferrine $(n=4)$ est majoritaire, mais seules les formes asialo- $(n=0)$, monosialo- $(n=1)$ et disialo-transferrine $(n=2)$ sont affectées à la hausse en cas d'exposition prolongée à l'éthanol, ce qui conduit davantage à la formation d'isoformes de la transferrine déficiente en carbohydrates (CDT ou formes avec 0 , 1 ou 2 résidus). Chez un sujet sobre, le taux de CDT est de l'ordre de $2 \%$, chiffre qui va rapidement augmenter lorsque la consommation d'éthanol pur est supérieure à $50 \mathrm{~g}$ par jour pendant au moins 8 jours. La demi-vie de la CDT est de l'ordre de 17 jours, ce qui en fait un index intéressant dans les cas d'alcoolisme intermittent. C'est un marqueur très sensible pour repérer la rechute chez des personnes alcoolo-dépendantes. Après 3 semaines d'imprégnation éthylique, le dosage de la CDT est normalisé [18, 23,24].

La sensibilité de ce test est estimée en moyenne à $70 \%$. C'est actuellement le marqueur de consommation excessive d'éthanol le plus performant. La spécificité de la CDT est supérieure à celle observée pour les marqueurs classiques, mais quelques facteurs sont susceptibles de conduire à une augmentation de la CDT, sans exposition à l'éthanol, comme les carences martiales, la grossesse, le syndrome de déficience en carbohydrates des glycoprotéines, qui est une maladie génétique rare et enfin certaines maladies hépatiques non-alcooliques [18].

Le dosage de la CDT est exclusivement sanguin. Il est tout à fait possible de réaliser des analyses sur du sang post mortem [25]. Différentes méthodes ont été proposées dans la littérature où dominent la chromatographie liquide, l'électrophorèse capillaire et l'immuno-chimie [24]. Il existe des kits de préparation (Ceofix CDT kit) facilitant l'approche en technique séparative.

Par immuno-chimie, il est possible de déterminer la CDT directement (Siemens N Latex CDT kit) ou après échange d'ions (Roche Tina Quant \%CDT ou Bio-Rad \%CDT TIA). Toutes les études [26,27] semblent indiquer que ces tests sont suffisamment robustes pour un usage en routine, mettant néanmoins en garde les biologistes quand les valeurs sont proches du seuil de positivité. Les techniques initiales, faisant appel à des traceurs radio-marqués sont de plus en plus abandonnées. 
Tableau I. Synthèse des marqueurs disponibles pour mettre en évidence une consommation d'alcool éthylique.

\begin{tabular}{|c|c|c|c|c|}
\hline Paramètre & Matrice(s) & Sensibilité & Spécificité & $\begin{array}{l}\text { Délai de } \\
\text { normalisation } \\
\text { après abstinence }\end{array}$ \\
\hline Éthanol & sang, urines, air expiré & $100 \%$ & $100 \%$ & qq heures \\
\hline \multirow[t]{3}{*}{ EtG } & sang & & $100 \%$ & qq heures \\
\hline & urines & & $100 \%$ & qq jours \\
\hline & cheveux & $\begin{array}{l}\text { variable selon le } \\
\text { seuil de positivité }\end{array}$ & $100 \%$ & $\begin{array}{l}\text { suivi par } \\
\text { segmentation }\end{array}$ \\
\hline EtS & cf EtG & cf EtG & cf EtG & cf EtG \\
\hline \multirow[t]{2}{*}{ FAEE } & cheveux & & & suivi par segmentation \\
\hline & méconium & & & - \\
\hline VGM & sang & $35-75 \%$ & $40-90 \%$ & 2 à 3 mois \\
\hline$\gamma$-GT & sérum & $35-90 \%$ & $35-75 \%$ & 15 jours \\
\hline CDT & sang, sérum & $50-95 \%$ & $80-100 \%$ & 3 semaines \\
\hline
\end{tabular}

\section{Et en pratique?}

De l'ensemble des considérations développées ci-dessus et à partir des opinions des différents auteurs, il apparaît qu'il n'existe pas de méthode unique, dotée d'une spécificité et d'une sensibilité à $100 \%$. Certains préconisent l'emploi de 2 approches simultanées, où la combinaison CDT- $\gamma$-GT peut être considérée comme très avantageuse.

Le tableau I reprend l'essentiel des données de la littérature (selon [18] et [23]).

Il convient également de déterminer d'emblée la situation clinique ou médico-légale qui motive les investigations. En effet, à chaque situation doit correspondre une réponse adaptée. Ainsi, l'auteur, à titre personnel, propose les approches suivantes :

- discrimination entre un buveur social et un buveur excessif : éthyl glucuronide dans les cheveux, avec un seuil conservateur à $50 \mathrm{pg} / \mathrm{mg}$;

- suivi de l'abstinence : CDT sérique, une fois par mois ;

- restitution du permis de conduire : CDT sérique et/ou éthyl glucuronide dans les cheveux ;

- dépistage à large échelle : $\gamma$-GT sérique ;

- examen post mortem : éthanolémie, CDT sérique et éthyl glucuronide dans le sang et les cheveux;

- recherche d'une exposition à l'éthanol dans une situation où sang et urine sont négatifs (augmentation de la fenêtre de détection, dans les affaires de soumission chimique par exemple) : éthyl glucuronide dans les urines.

\section{Conclusion}

Parfois qualifié de lubrifiant social, l'éthanol fait vivre en France des millions de personnes, mais a un coût social phénoménal. De très nombreuses pathologies, mais aussi des accidents et des implications médico-légales (trouble de l'ordre public, violences...) sont liés à une consommation excessive d'éthanol. Plusieurs marqueurs de l'éthylisme chronique ont été proposés dans la littérature. En plus de leur pertinence propre, s'ajoute le coût des analyses, fort différent selon les paramètres.

Cette revue a eu pour but de faire le point sur les différentes approches possible pour appréhender dans les meilleurs conditions la consommation excessive d'éthanol. La recherche n'a pas encore donné son mot de la fin, et d'autres paramètres sont en cours d'évaluation, comme le 5-hydroxytryptophol [28], le scyllo-inositol [29] ou encore une IgAs spécifique dirigée contre les adduits protéiques de l'acétaldéhyde [30].

\section{Références}

1. Beck F, Legleye S, Le Nézet O, Spilka S. Atlas régional des consommations d'alcool 2005. Données INPES/OFDT. Saint Denis : INPES, coll. Études santé territoires 2008.

2. SFTA. Alcools et glycols. Ann Toxicol Anal. 1999; numéro spécial: $1-92$.

3. Deveaux M. L'alcool. In : Mura P (coordonateur). Alcool, médicaments, stupéfiants et conduite automobile. Paris : Elsevier 1999 : 3-19.

4. Kamil IA, Smith NJ, Williams RT. A new aspect of ethanol metabolism : isolation of ethyl glucuronide. Biochem J. 1952; 51: 32-33.

5. Schmitt G, Droenner P, Skopp G, Aderjan R. Ethyl glucuronide concentrations in serum of human volunteers, teetotallers, and suspected drinking drivers. J Forensic Sci. 1997; 42: 10991102.

6. Wurst FM, Skipper GE, Weinmann W. Ethyl glucuronide-the direct ethanol metabolite on the threshold from science to routine use. Addiction. 2003; 98: 51-61.

7. Schloegel H, Rost T, Schmidt W, Wurst FM, Weinmann W. Distribution of ethyl glucuronide in rib bone marrow, other tissues and body liquids as proof of alcohol consumption before death. Forensic Sci Int. 2006; 156: 213-218.

8. Schummer C, Appenzeller B, Wenning R. Quantitative determination of ethyl glucuronide in sweat. Ther Drug Monit. 2008; 30: $536-539$. 
9. Kintz P, Villain M, Vallet E, Etter M, Salquebre G, Cirimele V. Ethyl glucuronide: unusual distribution between head hair and pubic hair. Forensic Sci Int. 2008; 176: 87-90.

10. Zimmer H, Schmitt G, Aderjan R. Preliminary immunochemical test for the determination of ethyl glucuronide in serum and urine: comparison of screening method result with gas chromatography-mass spectrometry. J Anal Toxicol. 2002; 26: 11-16.

11. Böttcher M, Beck O, Helander A. Evaluation of a new immunoassay for urinary ethyl glucuronide testing. Alcohol Alcohol. 2008; 43: 46-48.

12. Pragst F, Yegles M. Alcohol markers in hair. In : Kintz P (coordinateur). Analytical and Practical Aspects of Drug Testing in Hair. Boca Raton : CRC Taylor \& Francis, 2006 : 287-323.

13. Halter CC, Dresen S, Auwaerter V, Wurst FM, Weinmann W. Kinetics in serum and urinary excretion of ethyl sulfate and ethyl glucuronide after medium dose ethanol intake. Int J Leg Med. 2008; 122: 123-128.

14. Politi L, Morini L, Mari F, Groppi A, Bertol E. Ethyl glucuronide and ethyl sulfate in autopsy samples 27 years after death. Int J Leg Med. 2008; 122: 507-509.

15. Kaphalia BS, Cai P, Khan MF, Okorududu AO, Ansari GA. Fatty acid ethyl esters: markers of alcohol abuse and alcoholism. Alcohol. 2004; 34: 151-158.

16. Pragst F, Yegles M. Determination of fatty acid ethyl esters (FAEE) and ethyl glucuronide (EtG) in hair: a promising way for retrospective detection of alcohol abuse during pregnancy? Ther Drug Monit. 2008; 30: 255-263.

17. Gareri J, Lynn H, Handley M, Rao C, Koren G. Prevalence of fetal ethanol exposure in a regional population-based sample by meconium. Ther Drug Monit. 2008; 30: 239-245.

18. Vassault A, Fontaine H. Les marqueurs biologiques de l'alcoolisme. Rev Fr Lab. 2006; 387: 57-63.

19. Hansson P, Varga A, Krantz P, Alling C. Phosphatidylethanol in postmortem blood as a marker of previous heavy drinking. Int J Leg Med. 2001; 115: 158-161.

20. Varga A, Hansson P, Lundqvist C, Alling C. Phosphatidylethanol in blood as a marker of ethanol consumption in healthy volunteers: comparison with other markers. Alcohol Clin Exp Res. 1998; 22: 1832-1837.
21. Nalpas B, Vassault A, Charpin S, Lacour B, Berthelot P. Serum mitochondrial aspartate amino-transferase as a marker of chronic alcoholism: diagnosis value and interpretation in a liver unit. Hepathology. 1986; 6: 608-614.

22. Loguercio C, de Girolamo V, Cuomo A, Argenzio F, Iannotta C, Disalvo D, Grella A, del Vecchio Blanco C. Determination of plasma alpha-glutathione-S-tranferase in chronic alcohol abusers: relationship with alcohol intake and liver involvement. Alcohol Alcohol. 1998; 33: 366-372.

23. Donzé N, Augsburger M. L'évaluation de la consommation d'alcool. Caduceus Express. 2007; 9: 1.

24. Bortolotti F, De Paoli G, Tagliaro F. Carbohydrate-deficient transferrin (CDT) as a marker of alcohol abuse: a critical review of the literature 2001-2005. J Chromatogr B. 2006; 841: 96-109.

25. Simonnet C, Dumestre-Toulet V, Kintz P, Gromb S. Review of factors susceptible of influencing post-mortem carbohydratedeficient transferrin. Forensic Sci Int. 1999; 106: 7-17.

26. Bortolotti F, Tagliaro F, Cittadini F, Gottardo R, Trettene M, Marigo M. Determination of CDT, a marker of chronic alcohol abuse, for driving license issuing: immunoassay versus capillary electrophoresis. Forensic Sci Int. 2002; 128: 53-58.

27. Boehrer JL, Cano Y, Capolagui B, Desch G, Dosbaa I, Estepa L, Hennache B, Schellenberg F. Multisite validation of CDT measurement by the \%CDT TIA and the Tina Quant \%CDT kits. Ann Biol Clin. 2007; 65: 161-167.

28. Beck O, Stephanson N, Böttcher M, Dahmen N, Fehr C, Helander A. Biomarkers to disclose recent intake of alcohol: potential of 5-hydroxytryptophol glucuronide testing using new direct UPLC-tandem MS and ELISA methods. Alcohol Alcohol. 2007; 42: 321-325.

29. Viola A, Nicoli F, Denis B, Confort-Gouny S, Le Fur Y, Ranjeva JP, Viout P, Cozzone PJ. High cerebral scyllo-inositol: a new marker of brain metabolism disturbances induced by chronic alcoholism. MAGMA. 2004; 17: 47-61.

30. Hietala J, Koivisto H, Latvala J, Anttila P, Niemelä O. IgAs against acetaldehyde-modified red cell protein as a marker of ethanol consumption in male alcoholic subjects, moderate drinkers, and abstainers. Alcohol Clin Exp Res. 2006; 30: 1693-1698. 